\title{
Phylogenetic analysis of newly isolated grass carp reovirus
}

\author{
Xiu-ying Yan ${ }^{1}$, Ya Wang ${ }^{1}$, Ling-fang Xiong ${ }^{1}$, Ji-chang Jian ${ }^{1 *}$ and Zao-he $\mathrm{Wu}^{2}$
}

\begin{abstract}
Grass carp reovirus (GCRV) is a causative agent of haemorrhagic disease in grass carp that drastically affects grass carp aquaculture. Here we report a novel GCRV isolate isolated from sick grass carp that induces obvious cytopathic effect in CIK cells and name it as GCRV096. A large number of GCRV 096 viral particles were found in the infected CIK cells by electron microscope. The shape, size and the arrangement of this virus were similar to those of grass carp reovirus. With the primers designed according to GCRV 873 genome sequences, specific bands were amplified from sick grass carp and the infected CIK cells. The homology rates among vp4, vp6 and vp7 gene in GCRV 096 and those of some GCRV isolates were over $89 \%$. In this study, the sequences of $v p 4$, vp6 and $v p 7$ were used to analyse sequence variation, phylogenetic relationships and genotypes in twenty five GCRV isolates. The results indicated these twenty five GCRV isolates should be attributed to four genotypes. And there were no obvious characteristics in the geographical distribution of GCRV genotype. The study should provide the exact foundation for developing more effective prevention strategies of grass carp haemorrhagic disease.
\end{abstract}

Keywords: Grass carp reovirus (GCRV); Identification; Phylogenetic relationship; Genotype

\section{Introduction}

Grass carp reovirus (GCRV), which is known as a member of the Aquareovirus genus and the Reoviridae family, can cause serious haemorrhagic disease in grass carp (Chen and Jiang 1983) and obvious cytopathic effect (CPE) on many cell lines from fish (Zuo et al. 1986; Lu et al. 1990). To date, a number of various GCRV isolates have been isolated from diseased grass carp around the world, including GCRV 873, GCRV 875, GCRV HZ08, GCRV GD108, AGCRV and others (Fang et al. 2002; Chi et al. 2011; Ye et al. 2012; Zeng et al. 2013). These isolates are distinct not only in their levels of virulence and cell culture characteristics, but also in their antigenicity (Fang et al. 2002; Mohd Jaafar et al. 2008; Zhang et al. 2010a).

GCRV is a double-stranded RNA virus that is assigned to the Aquareovirus $C$ species. The genome of GCRV is known to consist of 11 segments of dsRNA contained in a core surrounded with a double-layered icosahedral capsid (Rangel et al. 1999). To our knowledge, there are few published reports about the serotype and genotype

\footnotetext{
* Correspondence: jianjc@gmail.com

'Guangdong Key Laboratory of Pathogenic Biology and Epidemiology for Aquatic Economic Animals, Guangdong Ocean University, Huguangyan East, Zhanjiang 524088, China

Full list of author information is available at the end of the article
}

of GCRV. Furthermore, there are no uniform criteria for virus genotyping. One of the virus genotyping methods is based on the analysis of the nucleotide sequence.

So far, some gene sequences of GCRV isolates have been reported (Mohd Jaafar et al. 2008; Rangel et al. 1999; Fang et al. 2000; Su et al. 2010; Attoui et al. 2002; Fan et al. 2010). $v p 4, v p 6$ and $v p 7$ gene in GCRV encode major outer capsid proteins and are conservative. Moreover, there are many variable sites and informative sites between sequences of $v p 4, v p 6$ and $v p 7$ gene in different GCRV isolates. Considering $v p 4, v p 6$ and $v p 7$ gene as molecular makers, we investigated sequence variation characteristics, the phylogenetic relationships and genotypes of twenty five GCRV isolates to find the evolutive characteristic of GCRV in the study. In this study, a new GCRV isolate was found and identified from diseased grass carp. This study provides the theoretical basis for the prevention and treatment of haemorrhagic disease in grass carp.

\section{Materials and methods \\ Virus and cells \\ GCRV 096 was isolated from the diseased grass carp in Xiaogan, Hubei Province and stored in our laboratory. A}


widely used GCRV sensitive cell steain, grass carp kidney cells (CIK) were purchased from shenzhen inspection and quarantine bureau in China. CIK is GCRV sensitive cell and are widely used in the related study of GCRV (Ye et al. 2012; Zhang et al. 2010b; Ma et al. 2011).

Some GCRV isolates were examined in the present study, which were identified in previous studies (Mohd Jaafar et al. 2008; Rangel et al. 1999; Fang et al. 2000; Su et al. 2010; Attoui et al. 2002; Fan et al. 2010). Table 1 presents information about the specific names of twenty five GCRV isolates, their abbreviations, locations where they were collected, the genes of GCRV and their GenBank accession numbers.

\section{Virus culture and transmission electron microscopy observation}

Cell culture, viral infection and propagation determination were performed as previously described (Fang et al. 1989). GCRV 096 particles were extracted with the differential centrifugation at 250-6000 g, and the supernatant was then ultracentrifuged at $35,000 \mathrm{~g}$ at $4^{\circ} \mathrm{C}$ for $2.5 \mathrm{~h}$. The purified virus pellet was resuspended in phosphatebuffered saline (PBS) with $\mathrm{pH} 7.4$ and then stored at $-70^{\circ} \mathrm{C}$ for the further use. After removing the cell culture medium from the confluent monolayer cell, the monolayer cell was rinsed two times with the PBS buffer and the virus was added with the adsorption for one hour at room temperature. Then, after aspirating off the virus, the maintain solution (M199 containing 2\% FBS) was added. The infected CIK cells were incubated at $28^{\circ} \mathrm{C}$ and observed daily.

Electron microscopic section of the infected CIK cells with CPE was made and observed in transmission electron microscope.

\section{RT-PCR amplification}

With viral RNA kit (Takara, Dalian, China), the GCRV 096 genome RNA was extracted from purified GCRV

Table 1 Names of GCRV isolates, abbreviations, localities, genes of GCRV used in this study and their GenBank accession numbers

\begin{tabular}{|c|c|c|c|c|}
\hline Names & Abbreviations & Localities & Genes & GenBank accession numbers \\
\hline AGCRV PB01-155 & 155 & America & $v p 4, v p 6, v p 7$ & EF589103, EF589105, EF589107 \\
\hline AGCRV & ARV & America & $v p 4, v p 6, v p 7$ & NC010589, NC010593, NC010594 \\
\hline GCRV 096 & 096 & Hubei, China & $v p 4, v p 6, v p 7$ & JN206664, HQ452490, JN206665 \\
\hline GCRV 104 & 104 & Hubei, China & $v p 6$ & HM234682 \\
\hline GCRV 097 & 097 & Shanxi, China & $v p 4$ & GQ469997 \\
\hline GCRV 873 & 873 & Hunan, China & $v p 4, v p 6, v p 7$ & AF403392, AF260512, AF260513, \\
\hline GCRV 875 & 875 & Hubei, China & vp6, vp7 & AF403412, AF403409 \\
\hline GCRV 876 & 876 & Jiangxi, China & $v p 6, v p 7$ & AF403413, AF403410 \\
\hline GCRV 991 & 991 & Hunan, China & $v p 6, v p 7$ & AF403414, AF403411 \\
\hline GCRV GD108 & 108 & Guangdong, China & $v p 4, v p 6, v p 7$ & HQ231208, HQ231205, HQ231203 \\
\hline GCRV HeNan988 & 988 & Henan, China & $v p 4, v p 6$ & KC847325, KC847328 \\
\hline GCRV HN12 & $\mathrm{H} 12$ & China & $v p 6$ & KC130075 \\
\hline GCRV HS11 & $\mathrm{H} 11$ & China & vp6 & KC130076 \\
\hline GCRV HuNan794 & 794 & Hunan, China & $v p 4, v p 6$ & KC238681, KC238684 \\
\hline GCRV HZO8 & H08 & Zhejiang, China & $v p 4, v p 6, v p 7$ & GQ896337, GU350746, GU350744 \\
\hline GCRV JS12 & $\mathrm{J} 12$ & China & vp6 & KC130077 \\
\hline GCRV NC11 & N11 & China & vp6 & KC130078 \\
\hline GCRV QC11 & Q11 & China & $v p 6$ & KC130079 \\
\hline GCRV QY12 & Q12 & China & $v p 6$ & KC130080 \\
\hline GCRV YX11 & Y11 & China & $v p 6$ & KC130081 \\
\hline GCRV ZS11 & $\mathrm{Z11}$ & China & vp6 & KC130082 \\
\hline GCRV 106 & 106 & China & $v p 4, v p 6$ & KC201171, KC201174 \\
\hline GCRV 918 & 918 & China & $v p 4, v p 6$ & KC201182, KC201185 \\
\hline GCRV JX01 & J01 & Jiangxi, China & $v p 7$ & JQ042807 \\
\hline GCRV JX02 & $\mathrm{J} 02$ & Jiangxi, China & $v p 7$ & $J \times 263303$ \\
\hline Bovine rotavirus B223 & Bovine & & $v p 4, v p 6, v p 7$ & D13394, AF317128, X57852 \\
\hline
\end{tabular}


096 virus. The cDNA of GCRV 096 genome RNA was acquired with RT-PCR kit (Takara, Dalian, China) using the random primers and M-MLV reverse transcriptase.

According to the genome sequence of GCRV 873, primers for GCRV $096 v p 4, v p 6$ and $v p 7$ gene amplification were designed based on homologous sequence in GCRV 873: 5" -CACTTCGCACTCTCTCTACAATG-3' and 5' -AGTACGACACTTCCCGCCGTT-3', 5' -TGTG ATGGCACAGCGTCAG-3' and 5'-GTTAGA CGAAC ATCGCCTGC-' 3 , 5' -TCACCACGATGCCACTTCAC$3^{\prime}$ and $5^{\prime}$-CGGTGCTTAATCGGATGGCT-3', respectively. Primers were also designed based on homologous sequence from GCRV GD108 and GCRV HZ08 for $v p 4$, $v p 6$ and $v p 7$ gene: $5^{\prime}$-ACTTACGGCCACTATCATGG$3^{\prime}$ and $5^{\prime}$-TCGGTGTACACGACCTAAG-3', 5' -CTTT GAGTCGACGCACGTAT-3' and 5' -CCGTCGGGTGG ATTAGGTC-3', 5'-TCTACTGCCAAGATGGCCAC-3' and $5^{\prime}$-GCACGCACCTTACTTACAGCA-3'. The PCR cycling conditions were an initial denaturation at $95^{\circ} \mathrm{C}$ for $3 \mathrm{~min}$ followed by 30 cycles consisting of $94^{\circ} \mathrm{C}$ for $30 \mathrm{~s}, 55^{\circ} \mathrm{C}$ for $60 \mathrm{~s}$ and $72^{\circ} \mathrm{C}$ for $70 \mathrm{~s}$, and a final extension step of $30 \mathrm{~min}$ at $72^{\circ} \mathrm{C}$. The composition of the PCR system $(50 \mu \mathrm{l})$ includes $33 \mu \mathrm{l}$ sterile water, $3 \mu \mathrm{l}$ $\mathrm{dNTP}$ (each is $2.5 \mathrm{mmol} / \mathrm{L}$ ), $10 \mathrm{pmol} / \mathrm{L}$ primer for $2 \mu \mathrm{l}$ each, $10 \times$ buffer for $5 \mu \mathrm{l}$ (containing $\mathrm{Mg}++$ ), DNA for $100 \mathrm{ng}$ and Taq polymerase for $0.25 \mu \mathrm{l}(5 \mathrm{U} / \mu \mathrm{l})$. The aimed genes were purified using Gel Extraction Kit (Takara, Dalian, China) from gelose gel and connected with pMD18-T vector at $16^{\circ} \mathrm{C}$, then transformed to DH5 $\alpha$ E.coli. The recombined plasmid was verified by sequencing.

\section{Gene sequence analysis of GCRV isolates}

Sequences of $v p 4, v p 6$ and $v p 7$ genes were aligned by using the Clustal V method in DNAstar software. Subsequently, the alignment was manually adjusted. Variable sites, information sites, genetic distances, and homologic rates of segments were calculated with MEGA5.1 (Tamura et al. 2007) and DnaSP5.10 (Rozas and Rozas 1999) software.

\section{Phylogenetic relationships of GCRV isolates}

Evolutionary models of $v p 4, v p 6$ and $v p 7$ gene in GCRV were separately simulated in ModelTest 3.7 (Posada and Crandall 1998). Subsequently, phylogenetic trees were restructured with simulation results. Using bovine rotavirus B223 as the outgroup, maximum parsimony (MP) trees, maximum likelyhood (ML) trees, and UPGMA trees were constructed with MEGA 4.1 (Tamura et al. 2007) software. MP trees were also built in PAUP4.0 (Swofford 1998) by running the heuristic search with TBR branch swapping, 100 random addition sequence replications, and non-parameter bootstrap resampling procedures to get the coincidence of the resultant MP trees. Bayesian analysis were performed with MrBayes3.12 (Huelsenbeck and Ronquist 2001) using the general-time-reversible + gamma + invariants (GTR + G + I) model of sequence evolution and four Markov Chain Monte Carlo (MCMC) sampling to assess phylogenetic relationships. We set the parameters in MrBayes as follows: $n s t=6$, rate $=$ gamma, basefreq $=$ estimate, generations $=10,000,000$, and the posterior probability and branches of the phylogeny were summed by burnin $=500$ and contype $=$ allcompat .

\section{Sequence variation analysis of $v p 4, v p 6$ and $v p 7$ genes in GCRV isolated to the same genotype}

Sequences of $v p 4, v p 6$ and $v p 7$ genes in GCRV isolated to the same genotype were aligned by using the Clustal V method in DNAstar software. Alignment was manually adjusted. Variable sites were analysed.

\section{Results}

Virus infection in sensitive cellls and particle identification Three days after the culture of the CIK cells infected by GCRV 096, CPE phenomenon was observed and the shedding and apoptosis occurred in most of the CIK cells five days after the infection. While, the controlled CIK cells without the infection by the virus grew well (Figure 1).

A large number of virus particles without the envelope structure crystalline in CIK cells were detected from transmission electron microscopy ultrathin section of CIK cells infected with GCRV 096 (Figure 1). The shape, size and the arrangement of GCRV 096 were similar to those of grass carp reovirus (Ke et al. 1990).

\section{Detection by RT-PCR}

$v p 4, v p 6$ and $v p 7$ genes were PCR amplified from GCRV 096, subcloned into a pMD18-T vector and sequenced. The length of $v p 4, v p 6$ and $v p 7$ genes in GCRV 096 was $1981 \mathrm{bp}, 1258 \mathrm{bp}$, and 855 bp, respectively (GenBank accession numbers: JN206664, HQ452490, and JN206665).

\section{Sequence analysis}

$v p 4, v p 6$ and $v p 7$ genes in the these GCRV isolates contain 184, 447 and 375 informative sites, respectively. Table 2 shows the identity and divergence among GCRV isolates based on $v p 4, v p 6$ and $v p 7$ genes, respectively. Based on the data shown in Table 2, it is apparent that genetic distances of $v p 4, v p 6$ and $v p 7$ genes among GCRV 096, GCRV 873, GCRV 875, GCRV JX01, GCRV 876 and GCRV 991 or AGCRV and AGVRV PB01-155 were small, and their homologous rates were high. Also, genetic distances among GCRV HZ08, GCRV GD108, GCRV 918, GCRV HuNan794, GCRV HeNan988, GCRV 106, GCRV ZS11, GCRV QC11, GCRV HN12, GCRV HS11, GCRV YX11, GCRV JS12, GCRV QY12, GCRV JX02, and GCRV 097 were small, with elevated homologous rates. 


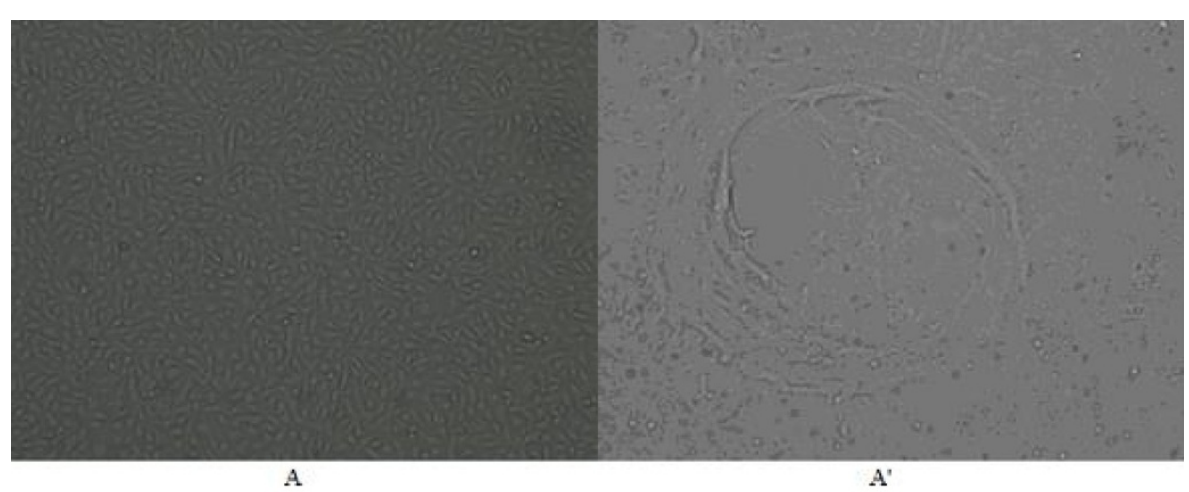

A. Uninfected cells

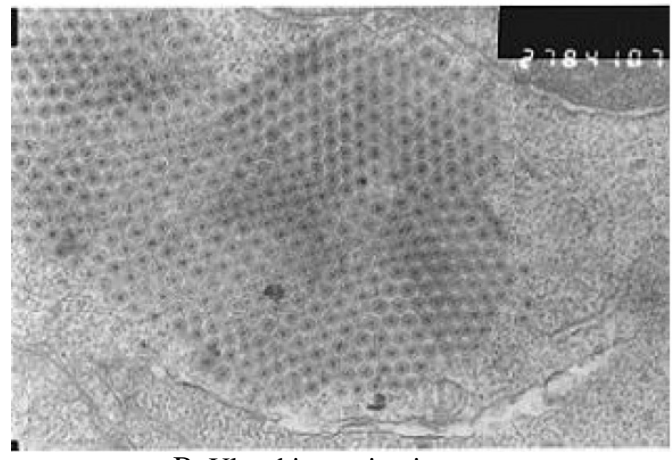

B. Ultrathin section image
A'. GCRV 096 infected CIK cells

Figure 1 CPE in the CIK cells $3 \mathrm{~d}$ after GCRV 096 isolate inoculation (A, $A^{\prime}$ 100x) and Crystalline array of viral particles (B 50,000x). Notes: A. The control CIK cells without GCRV096 inoculation. A'. CPE in the CIK cells 3 d after GCRV 096 isolate inoculation. B: Crystalline array of viral particles.

Other genetic distances were far and the genetic identities were small.

\section{Simulation results of evolutionary model and phylogenetic relationships of GCRV isolates}

Simulation results of the GCRV evolutionary model based on $v p 4, v p 6$ and $v p 7$ gene from ModelTest3.7 (Posada and Crandall 1998) are shown in Table 3. The simulation results are used to construct phylogenetic trees.

Topological structures of constructed phylogenetic trees, based on $v p 4, v p 6$ and $v p 7$ genes of GCRV in this article are basically coincident. According to evolutionary simulation results, there is the UPGMA tree constructed based on $v p 4$ gene in Figure 2. The results showed that the cluster on the top of the UPGMA tree consisted of GCRV 106, GCRV HeNan988, GCRV HuNan794, GCRV 097, GCRV 918, GCRV GD108 and GCRV HZ08. The second cluster was AGCRV PB01-155 and AGCRV. The third cluster contained GCRV 096 and GCRV 873.

In Figure 3, the MP tree was constructed based on $v p 6$ gene. On the MP tree, the cluster on the top consisted of GCRV 106, GCRV HeNan988, GCRV HuNan794, GCRV 918, GCRV ZS11, GCRV QC11, GCRV HN12, GCRV HS11, GCRV YX11, GCRV JS12, GCRV QY12, GCRV GD108 and GCRV HZ08. The second cluster was
GCRV 104. The next cluster was AGCRV PB01-155 and AGCRV. The last cluster contained GCRV 096, GCRV 875, GCRV 876, GCRV 991 and GCRV 873.

In Figure 4, the UPGMA tree was constructed based on $v p 7$ gene. The cluster on the top of this tree consisted of GCRV 096, GCRV 875, GCRV 876, GCRV JX01, GCRV 991 and GCRV 873. The second cluster was GCRV GD108, GCRV HZ08 and GCRV JX02. The last cluster contained AGCRV PB01-155 and AGCRV. The phylogenetic relationships of GCRV096, GCRV 991, GCRV 876, GCRV 873, GCRV JX01, and GCRV 875 or GCRV HZ08, GCRV JX02, and GCRV GD108 are relatively close.

\section{Sequence analysis of $v p 4, v p 6$ and $v p 7$ genes in GCRV isolated to the same genotype}

By analysing variable sites, we found the ratios of variation sites located on the third condon and transitions were respectively $71.6 \%$ and $82.1 \%$ in $v p 4$ gene; $57.1 \%$ and $80.0 \%$ in $v p 6$ gene; $77.3 \%$ and $89.3 \%$ in $v p 7$ gene.

\section{Discussion}

Amongst all aquareovirus isolates, GCRV is one of the most pathogenic agents (Fang et al. 2002). GCRV can cause fatal epidemics of haemorrhagic disease in grass carp, and affects approximately $85 \%$ of fingerling and 
Table 2 Identity (above the diagonal) and divergence (under the diagonal) between GCRV isolates based on the vp4, vp6, vp7 gene [ $\times 1000]$

\begin{tabular}{|c|c|c|c|c|c|c|c|c|c|c|c|c|c|c|c|c|c|c|c|c|c|c|c|}
\hline \multirow[b]{2}{*}{ GCRV } & \multicolumn{11}{|c|}{ Based on the vp4 gene } & \multicolumn{12}{|c|}{ Based on the $v p 7$ gene } \\
\hline & 155 & ARV & 096 & 097 & 873 & 108 & 988 & 794 & H08 & 106 & 918 & GCRV & 155 & AVR & 096 & 873 & 875 & 876 & 991 & 108 & H08 & J01 & J02 \\
\hline 155 & & 1000 & 599 & 317 & 603 & 299 & 293 & 292 & 286 & 293 & 296 & 155 & & 1000 & 203 & 303 & 279 & 287 & 289 & 219 & 214 & 302 & 213 \\
\hline ARV & 0 & & 599 & 317 & 603 & 299 & 293 & 292 & 286 & 293 & 296 & ARV & 0 & & 203 & 303 & 279 & 287 & 289 & 219 & 214 & 302 & 213 \\
\hline 096 & 453 & 453 & & 295 & 993 & 303 & 299 & 298 & 304 & 298 & 304 & 096 & 1178 & 1178 & & 212 & 196 & 197 & 197 & 199 & 208 & 213 & 207 \\
\hline 097 & 946 & 946 & 827 & & 311 & 981 & 990 & 987 & 994 & 990 & 987 & 873 & 767 & 767 & 920 & & 902 & 999 & 1000 & 199 & 197 & 994 & 205 \\
\hline 873 & 445 & 445 & 5 & 827 & & 298 & 297 & 296 & 299 & 296 & 302 & 875 & 856 & 856 & 910 & 100 & & 901 & 902 & 222 & 208 & 899 & 206 \\
\hline 108 & 967 & 967 & 886 & 20 & 872 & & 970 & 970 & 963 & 971 & 968 & 876 & 776 & 776 & 855 & 1 & 102 & & 999 & 204 & 200 & 993 & 197 \\
\hline 988 & 971 & 971 & 891 & 10 & 882 & 31 & & 997 & 986 & 998 & 986 & 991 & 782 & 782 & 848 & 0 & 100 & 1 & & 204 & 200 & 995 & 195 \\
\hline 794 & 977 & 977 & 896 & 13 & 888 & 31 & 3 & & 985 & 998 & 985 & 108 & 1218 & 1218 & 920 & 885 & 891 & 865 & 870 & & 986 & 203 & 987 \\
\hline H08 & 941 & 941 & 875 & 6 & 863 & 35 & 14 & 14 & & 986 & 984 & H08 & 1194 & 1194 & 904 & 863 & 880 & 843 & 848 & 14 & & 202 & 998 \\
\hline 106 & 973 & 973 & 893 & 10 & 884 & 30 & 2 & 2 & 13 & & 987 & J01 & 772 & 772 & 897 & 6 & 103 & 7 & 5 & 890 & 864 & & 193 \\
\hline 918 & 962 & 962 & 897 & 13 & 888 & 33 & 15 & 15 & 17 & 13 & & J02 & 1300 & 1300 & 913 & 858 & 876 & 839 & 844 & 14 & 2 & 859 & \\
\hline
\end{tabular}

\begin{tabular}{|c|c|c|c|c|c|c|c|c|c|c|c|c|c|c|c|c|c|c|c|c|c|c|}
\hline \multicolumn{23}{|c|}{ Based on the $v p 6$ gene } \\
\hline$\overline{\text { GCRV }}$ & 155 & ARV & 096 & 104 & 873 & 875 & 876 & 991 & 108 & \multirow{2}{*}{$\begin{array}{l}988 \\
233\end{array}$} & \multirow{2}{*}{$\begin{array}{l}\mathrm{H} 12 \\
241\end{array}$} & \multirow{2}{*}{$\begin{array}{l}\mathrm{H} 11 \\
235\end{array}$} & \multirow{2}{*}{$\begin{array}{l}794 \\
233\end{array}$} & H08 & $\mathrm{J} 12$ & N11 & Q11 & Q12 & Y11 & Z11 & 106 & 918 \\
\hline 155 & & 1000 & 548 & 230 & 548 & 547 & 547 & 548 & 196 & & & & & 204 & 235 & 235 & 235 & 235 & 235 & 233 & 233 & 232 \\
\hline ARV & 0 & & 548 & 230 & 548 & 547 & 547 & 548 & 196 & 233 & 241 & 235 & 233 & 204 & 235 & 235 & 235 & 235 & 235 & 233 & 233 & 232 \\
\hline 096 & 500 & 500 & & 220 & 994 & 998 & 998 & 997 & 201 & 242 & 235 & 235 & 241 & 202 & 233 & 233 & 239 & 233 & 233 & 243 & 241 & 241 \\
\hline 104 & 936 & 936 & 769 & & 238 & 246 & 246 & 246 & 190 & 232 & 229 & 232 & 232 & 198 & 238 & 238 & 228 & 238 & 232 & 232 & 232 & 223 \\
\hline 873 & 503 & 503 & 6 & 751 & & 999 & 999 & 998 & 204 & 243 & 235 & 235 & 242 & 205 & 232 & 232 & 239 & 232 & 232 & 243 & 242 & 242 \\
\hline 875 & 516 & 516 & 3 & 751 & 1 & & 1000 & 999 & 211 & 238 & 231 & 231 & 238 & 203 & 233 & 233 & 236 & 233 & 233 & 238 & 238 & 237 \\
\hline 876 & 516 & 516 & 3 & 751 & 1 & 0 & & 999 & 211 & 238 & 231 & 231 & 238 & 203 & 233 & 233 & 236 & 233 & 233 & 238 & 238 & 237 \\
\hline 991 & 514 & 514 & 4 & 755 & 2 & 1 & 1 & & 211 & 238 & 231 & 236 & 238 & 200 & 233 & 233 & 236 & 233 & 233 & 238 & 238 & 237 \\
\hline 108 & 1427 & 1427 & 1340 & 1527 & 1261 & 1236 & 1236 & 1236 & & 207 & 197 & 203 & 207 & 965 & 204 & 204 & 203 & 204 & 197 & 200 & 200 & 199 \\
\hline 988 & 834 & 834 & 682 & 939 & 693 & 665 & 665 & 665 & 1157 & & 975 & 972 & 998 & 208 & 971 & 971 & 990 & 971 & 973 & 998 & 998 & 995 \\
\hline $\mathrm{H} 12$ & 844 & 844 & 680 & 959 & 692 & 659 & 659 & 659 & 1214 & 26 & & 991 & 975 & 210 & 990 & 990 & 979 & 990 & 992 & 975 & 976 & 973 \\
\hline H11 & 850 & 850 & 683 & 962 & 694 & 662 & 662 & 662 & 1211 & 29 & 9 & & 973 & 205 & 998 & 998 & 981 & 998 & 999 & 973 & 974 & 971 \\
\hline 794 & 838 & 838 & 685 & 931 & 697 & 670 & 670 & 670 & 1149 & 2 & 25 & 28 & & 206 & 972 & 972 & 990 & 972 & 974 & 998 & 999 & 996 \\
\hline H08 & 1400 & 1400 & 1288 & 1510 & 1210 & 1196 & 1196 & 1196 & 32 & 1113 & 1165 & 1162 & 1106 & & 204 & 204 & 200 & 204 & 205 & 206 & 206 & 204 \\
\hline $\mathrm{J} 12$ & 850 & 850 & 683 & 958 & 694 & 662 & 662 & 662 & 1228 & 29 & 10 & 2 & 29 & 1178 & & 1000 & 980 & 1000 & 998 & 972 & 973 & 970 \\
\hline N11 & 850 & 850 & 683 & 958 & 694 & 662 & 662 & 662 & 1228 & 29 & 10 & 2 & 29 & 1178 & 0 & & 980 & 1000 & 998 & 972 & 973 & 970 \\
\hline Q11 & 834 & 834 & 689 & 923 & 701 & 670 & 670 & 670 & 1142 & 10 & 22 & 19 & 10 & 1099 & 20 & 20 & & 980 & 982 & 992 & 991 & 988 \\
\hline Q12 & 850 & 850 & 683 & 958 & 694 & 662 & 662 & 662 & 1228 & 29 & 10 & 2 & 29 & 1178 & 0 & 20 & 20 & & 988 & 972 & 973 & 970 \\
\hline
\end{tabular}


Table 2 Identity (above the diagonal) and divergence (under the diagonal) between GCRV isolates based on the vp4, vp6, vp7 gene [ $\times 1000]$ (Continued)

\begin{tabular}{|c|c|c|c|c|c|c|c|c|c|c|c|c|c|c|c|c|c|c|c|c|c|c|}
\hline Y11 & 854 & 854 & 686 & 958 & 698 & 667 & 667 & 667 & 1219 & 28 & 8 & 1 & 27 & 1170 & 2 & 2 & 19 & 2 & & 974 & 975 & 971 \\
\hline Z11 & 834 & 834 & 682 & 939 & 693 & 665 & 665 & 665 & 1142 & 2 & 25 & 28 & 2 & 1099 & 29 & 29 & 8 & 29 & 27 & & 999 & 996 \\
\hline 106 & 838 & 838 & 685 & 935 & 697 & 670 & 670 & 670 & 1149 & 2 & 24 & 27 & 1 & 1106 & 28 & 28 & 9 & 28 & 26 & 1 & & 997 \\
\hline 918 & 851 & 851 & 692 & 946 & 704 & 677 & 677 & 677 & 1154 & 5 & 28 & 30 & 4 & 1124 & 31 & 31 & 12 & 31 & 29 & 4 & 3 & \\
\hline
\end{tabular}


Table 3 Simulation results of the evolutionary model

\begin{tabular}{|c|c|c|c|c|c|}
\hline & & & vp4 gene & vp6 gene & vp7 gene \\
\hline Model selected: & $H K Y+G$ & $\mathrm{GTR}+\mathrm{G}$ & $\mathrm{HKY}+\mathrm{G}$ & TVMef $+\mathrm{G}$ & $\mathrm{K} 80+\mathrm{G}$ \\
\hline$-\operatorname{lnL}=$ & 10960.6680 & 10947.8525 & 10486.8574 & 10480.6113 & 6139.4121 \\
\hline$K=$ & 5 & 9 & 5 & 8 & 2 \\
\hline $\mathrm{AIC}=$ & & 21913.7051 & & & 20977.2227 \\
\hline \multicolumn{6}{|l|}{ Base frequencies: } \\
\hline freq $A=$ & 0.2726 & 0.2724 & 0.2561 & 0.2530 & \\
\hline freq $C=$ & 0.2541 & 0.2476 & 0.2612 & 0.2586 & \\
\hline freq $G=$ & 0.2361 & 0.2401 & 0.2285 & 0.2313 & \\
\hline freq $\top=$ & 0.2371 & 0.2400 & 0.2541 & 0.2571 & \\
\hline \multicolumn{6}{|l|}{ Substitution model: } \\
\hline $\mathrm{R}(\mathrm{a})[\mathrm{A}-\mathrm{C}]=$ & & 1.9847 & & 1.8471 & \\
\hline$R(b)[A-G]=$ & & 3.8446 & & 7.0961 & \\
\hline $\mathrm{R}(\mathrm{c})[\mathrm{A}-\mathrm{T}]=$ & & 1.3223 & & 1.3157 & \\
\hline $\mathrm{R}(\mathrm{d})[\mathrm{C}-\mathrm{G}]=$ & & 1.7384 & & 1.3664 & \\
\hline $\mathrm{R}(\mathrm{e})[\mathrm{C}-\mathrm{T}]=$ & & 4.4150 & & 7.0961 & \\
\hline$R(f)[G-T]=$ & & 1.0000 & & 1.0000 & \\
\hline $\mathrm{i} / \mathrm{tv}$ ratio $=$ & 1.3563 & & 2.5439 & & \\
\hline \multicolumn{6}{|c|}{ Proportion of invariable } \\
\hline sites $=$ & 0 & 0 & 0 & 0 & 0 \\
\hline \multicolumn{6}{|l|}{ Gamma distribution } \\
\hline shape parameter $=$ & 4.2112 & 4.3849 & 3.8529 & 3.7611 & 3.8868 \\
\hline
\end{tabular}

yearling populations (Jiang and Ahne 1989). Many GCRV isolates have been isolated in recent years, and various of them have been reported to exhibit distinctive differences in virulence (Fang et al. 2002). Moreover, new GCRV isolates were found constantly. In this study, GCRV 096 is a new GCRV isolate similar to GCRV 873, GCRV 875, GCRV 876, GCRV 991 and GCRV JX01.

In order to analyse the difference among GCRV isolates as well as their evolutionary relatiohship, it is necessary to genotyping. Currently, uniform criteria in place for virus genotyping are still unavailable. In hepatitis $\mathrm{C}$ virus, a more than $30 \%$ nucleotide sequence divergence between genotypes is generally considered standard (Simmonds 2004). The genetic heterogeneity among genotypes of hepatitis $\mathrm{E}$ virus has been shown to be more than $20 \%$ (Schlauder and Mushahwar 2001). In GCRV, relatively conservative $v p 4, v p 6$ and $v p 7$ gene encode major outer capsid proteins and consist of many variable sites (Rangel et al. 1999). So, $v p 4, v p 6$ and $v p 7$ gene could be used for GCRV genotyping.

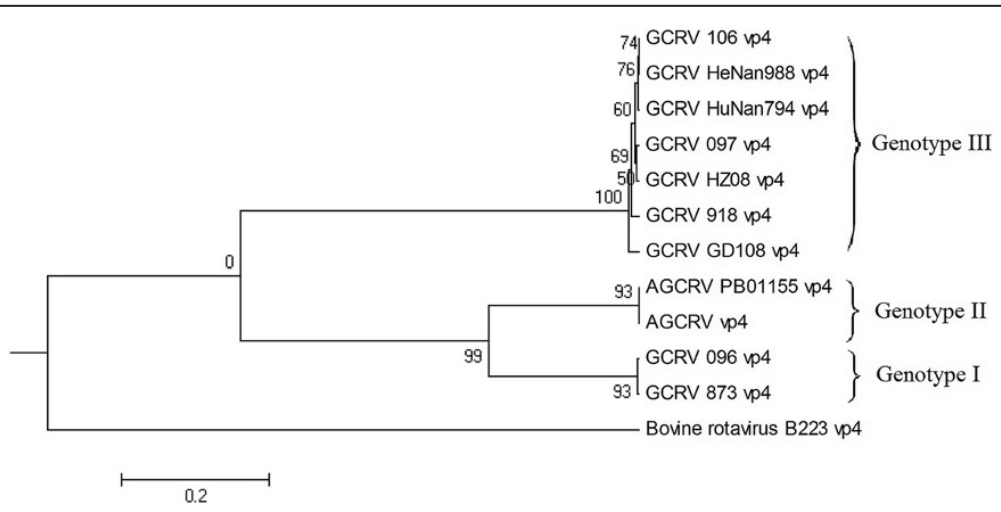

Figure 2 The constructed UPGMA tree based on the $v p 4$ gene (Numbers indicate degree of confidence) was created first in MEGA software and completed with Microsoft Paint program. 


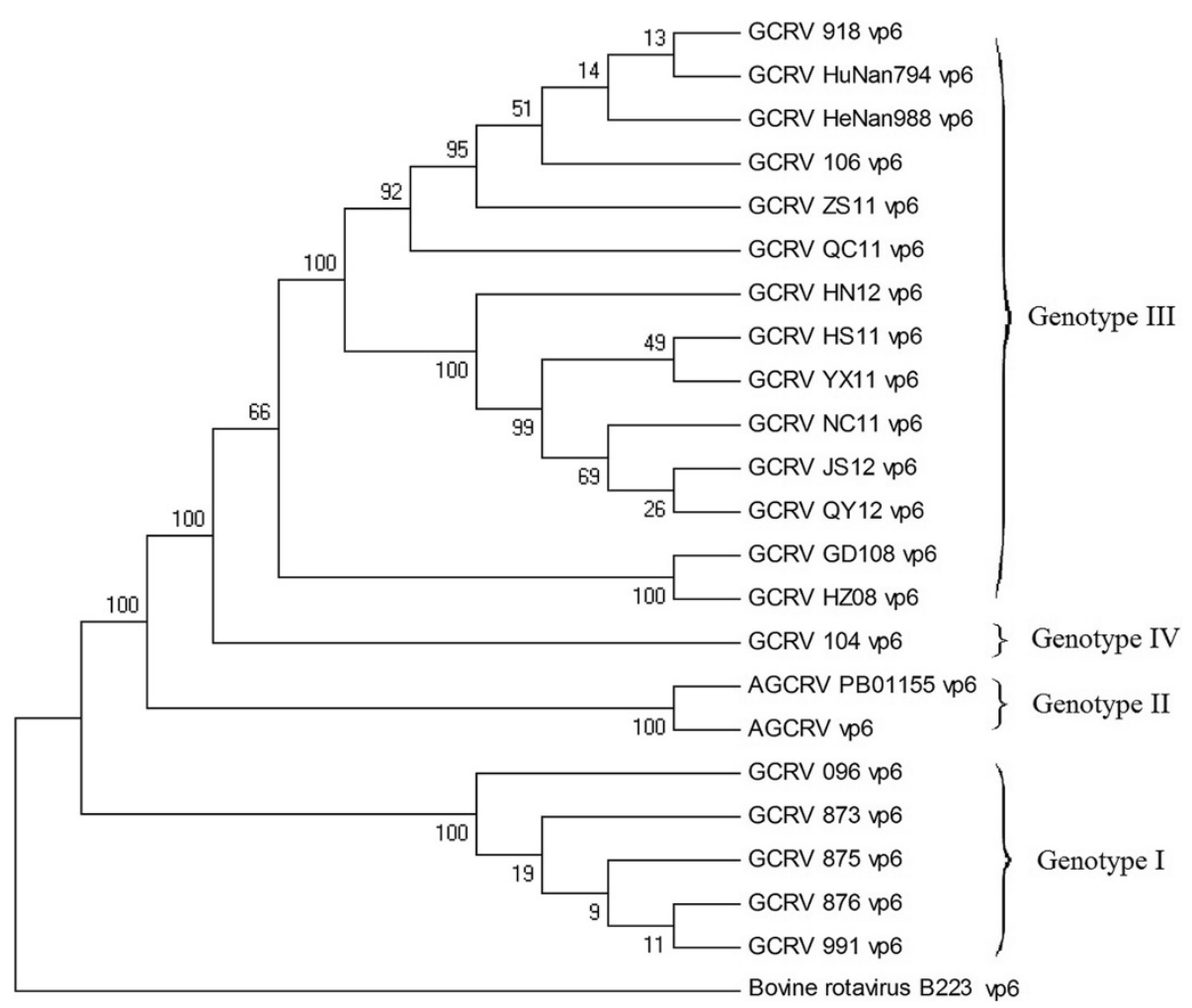

Figure 3 The constructed MP tree based on the vp6 gene (Numbers indicate the degree of confidence) was created first in PAUP software and completed with Microsoft Paint program.

The genetic distances among GCRV 096, GCRV JX01, GCRV 873, GCRV 875, GCRV 876 and GCRV 991 were small with high homologous rates. Furthermore, these isolates clustered together into one cluster on constructed phylogenetic trees. These results present that GCRV 096, GCRV JX01, GCRV 873, GCRV 875, GCRV 876 and GCRV 991 are attributed to the same genotype, i.e. genotype I. Genetic distances between AGCRV PB01-155 and AGVRV were small and their homologous rates were also high. On phylogenetic trees, AGCRV and AGCRV
PB01-155 separately clustered into one cluster. These results indicate that AGCRV and AGCRV PB01-155 are attributed to a new genotype, i.e. genotype II. Genetic distances among GCRV HZ08, GCRV GD108, GCRV 918, GCRV HuNan794, GCRV HeNan988, GCRV 106, GCRV ZS11, GCRV QC11, GCRV HN12, GCRV HS11, GCRV YX11, GCRV JS12, GCRV QY12, GCRV JX02, and GCRV 097 were extremely small with especially high homologous rates. Furthermore, these isolates clustered together into one cluster on phylogenetic trees. GCRV

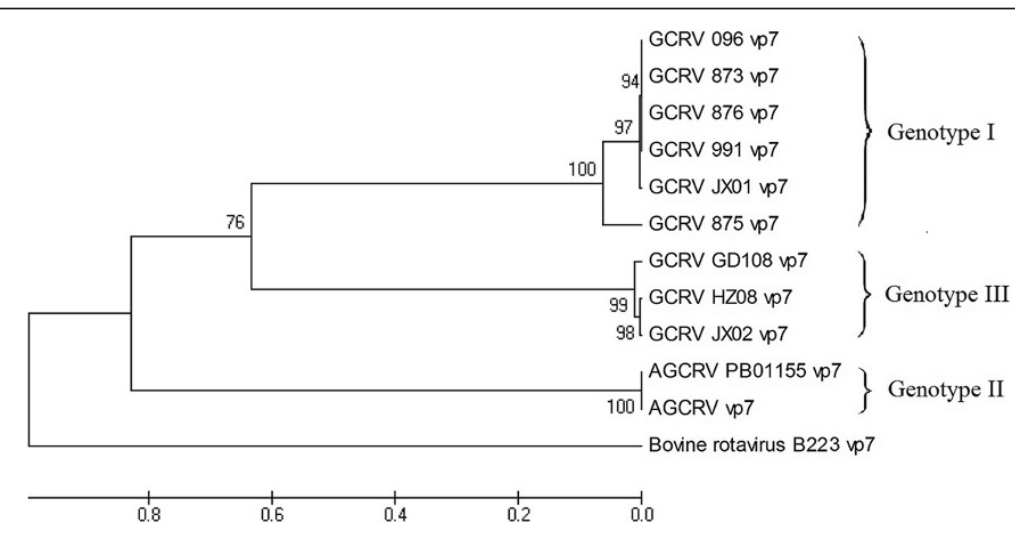

Figure 4 The constructed UPGMA tree based on the vp7 gene (Numbers indicate the degree of confidence) was created first in MEGA software and completed with Microsoft Paint program. 
HZ08, GCRV GD108, GCRV 918, GCRV HuNan794, GCRV HeNan988, GCRV 106, GCRV ZS11, GCRV QC11, GCRV HN12, GCRV HS11, GCRV YX11, GCRV JS12, GCRV QY12, GCRV JX02, and GCRV 097 were attributed to another new genotype, i.e. genotype III. In contrast, genetic distances between GCRV 104 and other GCRV isolates were large, and their homologous rates were small. On the phylogenetic tree (Figure 4), GCRV 104 separately clustered into one cluster. GCRV 104 is attributed to a new genotype, i.e. genotype IV.

The genotyping results obtained are consistent with previous research conclusions. The study of Wang indicated there were different genotypes of GCRV in China (Wang et al. 2012a). The biological characteristics of GCRV isolates belonging to the same genotype indicated they were analogous. For example, in an artificial infection test, GCRV HZ08 and GCRV GD108 can cause mortality of $60-80 \%$ of the yearly grass carp (approx. $10 \mathrm{~cm}$ in length), without obvious CPE in CIK cells (Ye et al. 2012; Zhang et al. 2010b). However, American grass carp reovirus (AGCRV) is not strongly connected with infectious disease in fish, although it is commonly detected by cell culture during routine inspections of healthy fish (Goodwin et al. 2010). GCRV 873, GCRV 096, GCRV 875, GCRV 876, GCRV 991 and GCRV JX01 can arouse significant CPE in CIK cells (Zhang et al. 2010a; Wang et al. 2012b). Furthermore, other characteristics of these two isolates were also similar. The genomic segments pattern of GCRV 875 was found to be similar to that of GCRV 873 (Fang et al. 2002). Polyacrylamide gel electrophoresis atlases of GCRV 873, GCRV 875, GCRV 876 and GCRV 991 were also the same (Fang et al. 2002).

The comparative analysis of the geographic location (Table 1) of collected GCRV isolates together with the difference between GCRV isolates and GCRV genotyping indicated there was no obvious relationship between the evolution of GCRV and geographical distribution of GCRV. In the same genotype, the ratios of variation sites on the third condon and the transitions in $v p 4, v p 6$ and $v p 7$ gene were high.

Hemorragic disease of grass carp outbreaks seriously in China. Many isolates of grass carp reovirus have been discovered while new isolates are being isolated constantly. The systematic difference comparison of the different GCRV isolates has not been reported. In this study, we have verified the diference among various GCRV genotypes. GCRV genotyping has important significance to diagnosis and treatment in hemorrhagic disease of grass carp, especially to vaccine development. Comparison of different GCRV isolates and genotyping are helpful to further our understanding in GCRV genetic variation and evolution and the development of more effective preventative strategies against GCRV.
This study provides a foundation for revealing differences among GCRV isolates. Simultaneously, it is significant for the further research on genetically engineered vaccines against grass carp haemorrhagic disease and grass carp breeding for disease resistance.

\section{Abbreviation}

bp: Base pairs; CIK: Grass carp kidney cell line; CPE: Cytopathic effect; dNTPs: Deoxynucleotide triphosphates; FBS: Fetal bovine serum; GCRV: Grass carp reovirus; $\mathrm{MgCl}_{2}$ : Magnesim chloride; min: Minute; ML: Maximum likelihood; MP: Maximum parsimony; PBS: Phosphate-buffered saline; PCR: Polymerase chain reaction; RT-PCR: Reverse transcription-PCR; TBR: Tree-bisection-recorrnection.

\section{Competing interests}

The authors declare that they have no competing interests. The authors alone are responsible for the content and writing of the paper.

\section{Authors' contributions}

YXY, JJC and WZH conceived and designed the experiments described in this work and wrote the manuscript. YXY, WY and XLF performed and analyzed the data. JJC and WZH supervised the work and analyzed the results. All the authors read and approved the final manuscript.

\section{Acknowledgements}

The study was supported by the National 973 Plan Project in China (No. 2009CB118704). The authors would like to thank Dr. JY Shen for her help.

\section{Author details}

'Guangdong Key Laboratory of Pathogenic Biology and Epidemiology for Aquatic Economic Animals, Guangdong Ocean University, Huguangyan East, Zhanjiang 524088, China. ${ }^{2}$ Zhongkai University of Agriculture and

Engineering, Guangzhou 510225, China.

Received: 13 January 2014 Accepted: 27 March 2014

Published: 15 April 2014

\section{References}

Chen YS, Jiang YL (1983) Morphology and physical and chemical properties of grass carp hemorrhage virus. KeXue Tong-Bao 18:1138-1140. In Chinese

Zuo WG, Qian HX, Xu YF, Du SY, Yang XL (1986) A cell line derived from the kidney of grass carp (ctenopharyngodon idellus). J Fish China 10(1):11-17. In Chinese

Lu YA, Lannan CN, Rohovec JS, Fryer JL (1990) Fish cell lines: establishment and characterization of three new cell lines from grass carp (Ctenopharyngodon idella). Vitro Cell Dev Biol 26(3 Pt 1):275-279

Fang Q, Xiao TY, Li L, Zou GP, Zhang HY, Wang YP (2002) Infection Characterizations of four Grass Carp Reovirus (GCRV) strains. Virol Sin 17:182-184. In Chinese

Chi YY, Tian YY, Ye X, Deng GC, Li J, Wang HJ (2011) Molecular properties of grass carp reovirus in southern China and establishment of duplex PCR detection method. Bing Du Xue Bao 27(4):358-365

Ye X, Tian YY, Deng GC, Chi YY, Jiang XY (2012) Complete genomic sequence of a reovirus isolated from grass carp in China. Virus Res 163(1):275-283

Zeng WW, Wang Q, Wang YY, Xu DH, Wu SQ (2013) A one-step molecular biology method for simple and rapid detection of grass carpCtenopharyngodon idella reovirus (GCRV) HZ08 strain. J Fish Biol 82(5):1545-1555

Mohd Jaafar F, Goodwin AE, Belhouchet M, Merry G, Fang Q, Cantaloube JF, Biagini P, de Micco P, Mertens PPC, Attoui H (2008) Complete characterisation of the American grass carp reovirus genome (genus Aquareovirus: family Reoviridae) reveals an evolutionary link between aquareoviruses and coltiviruses. Virology 373(2):310-321

Zhang LL, Luo Q, Fang Q, Wang YP (2010a) An improved RT-PCR assay for rapid and sensitive detection of grass carp reovirus. J Virol Methods 169(1):28-33

Rangel AA, Rockemann DD, Hetrick FM, Samal SK (1999) Identification of grass carp haemorrhage virus as a new genogroup of aquareovirus. J Gen Virol 80(9):2399-2402

Fang Q, Attoui H, Cantaloube JF, Biagini P, Zhu ZY, De Micco P, De Lamballerie X (2000) Sequence of genome segments 1, 2, and 3 of the grass carp reovirus 
Su JG, Huang T, Dong J, Heng JF, Zhang RF, Peng LM (2010) Molecular cloning and immune responsive expression of MDA5 gene, a pivotal member of the RLR gene family from grass carp Ctenopharyngodon idella. Fish Shellfish Immun 28(4):712-718

Attoui H, Fang Q, Mohd Jaafar F, Cantaloube JF, Biagini P, de Micco P, de Lamballerie $X$ (2002) Common evolutionary origin of aquareoviruses and orthoreoviruses revealed by genome characterization of Golden shiner reovirus, Grass carp reovirus, Striped bass reovirus and golden ide reovirus (genus Aquareovirus, family Reoviridae). J Gen Virol 83(8):1941-1951

Fan C, Shao L, Fang Q (2010) Characterization of the nonstructural protein NS80 of grass carp reovirus. Arch Virol 155(11):1755-1763

Zhang C, Wang Q, Shi CB, Zeng WW, Liu Y, Wu SQ (2010b) Molecular analysis of grass carp reovirus HZ08 genome segments 1-3 and 5-6. Virus Genes 41(1):102-104

Ma J, Wang W, Zeng L, Fan Y, Xu J, Zhou Y (2011) Inhibition of the replication of grass carp reovirus in CIK cells with plasmid-transcribed shRNAs. J Virol Methods 175(2):182-187

Fang Q, Ke LH, Cai YQ (1989) Growth characteristics and high titer culture of grass carp hemorrhage virus (GCHV)-873 in vitro. Virol Sinion 3:314-319. In Chinese

Tamura K, Dudley J, Nei M, Kumar S (2007) MEGA4: molecular evolutionary genetics analysis (MEGA) software version 4.0. Mol Biol Evol 24(8):1596-1599

Rozas J, Rozas R (1999) DnaSP version 3: an integrated program for molecular population genetics and molecular evolution analysis. Bioinformatics 15(2):174-175

Posada D, Crandall K (1998) Modeltest: testing the model of DNA substitution. Bioinformatics 14(9):817-818

Swofford DL (1998) PAUP: Phylogenetic analysis using parsimony and other methods Associates. Sinauer, Sunderland, M A

Huelsenbeck JP, Ronquist F (2001) MrBayes: Bayesian inference of phylogenetic trees. Bioinformatics 17(8):754-755

Ke LH, Fang Q, Cai YQ (1990) Characteristics of a novel isolate of grass carp hemorrhagic virus. ACTA Hydrobiol Sin 14(2):153-159. In Chinese

Jiang Y, Ahne W (1989) Some properties of the etiological agent of the hemorrhagic disease of grass carp and black carp. In: Ahne W, Kurstak E (ed) Viruses of Lower Vertebrates. Springer-Verlag, Berlin, pp 227-239

Simmonds P (2004) Genetic diversity and evolution of hepatitis $C$ virus-15 years on. J Gen Virol 85(11):3173-3188

Schlauder GG, Mushahwar IK (2001) Genetic heterogeneity of hepatitis E virus. J Med Virol 65(2):282-292

Wang Q, Zeng WW, Liu C, Zhang C, Wang YY, Shi CB, Wu SQ (2012a) Complete genome sequence of a reovirus isolated from grass carp, indicating different genotype of GCRV in China. J Virol 86(22):12466

Goodwin AE, Merry GE, Attoui H (2010) Detection and prevalence of the nonsyncytial American grass carp reovirus Aquareovirus $\mathrm{G}$ by quantitative reverse transcriptase polymerase chain reaction. J Aquat Anim Health 22(1):8-13

Wang T, Xu D, Lv LQ (2012b) Detection of the co-infection of different grass carp reovirus strains using dsRNA sequencing technology. J Shanghai Ocean Univ 21(5):756-762. In Chinese

\section{doi:10.1186/2193-1801-3-190}

Cite this article as: Yan et al:: Phylogenetic analysis of newly isolated grass carp reovirus. SpringerPlus 2014 3:190.

\section{Submit your manuscript to a SpringerOpen ${ }^{\circ}$ journal and benefit from:}

- Convenient online submission

- Rigorous peer review

- Immediate publication on acceptance

- Open access: articles freely available online

- High visibility within the field

- Retaining the copyright to your article

Submit your next manuscript at $\gg$ springeropen.com 